\title{
Gender Differences in Haptic Parallelity Matching
}

\author{
Hanneke I. Van Mier \\ Department of Cognitive Neuroscience, Faculty of Psychology and Neuroscience, Maastricht University, Maastricht, \\ The Netherlands \\ Email: h.vanmier@maastrichtuniversity.nl
}

How to cite this paper: Van Mier, H. I. (2021). Gender Differences in Haptic Parallelity Matching. Psychology, 12, 607-623. https://doi.org/10.4236/psych.2021.124038

Received: January 25, 2021

Accepted: April 25, 2021

Published: April 28, 2021

Copyright (c) 2021 by author(s) and Scientific Research Publishing Inc. This work is licensed under the Creative Commons Attribution International License (CC BY 4.0).

http://creativecommons.org/licenses/by/4.0/

\begin{abstract}
Previous studies have reported gender differences in performance when two bars have to be set parallel to each other haptically, with females having significantly larger deviations than males. Recent results comparing male participants with and without action video game experience showed that the former performed significantly better than the latter in the aforementioned haptic parallelity task. Considering that males more often engage in action video gaming, the observed performance differences between male and female participants might be related to gaming experience rather than gender. This was investigated in the current study by comparing haptic parallelity performance in males with action video gaming experience and in males and females without this experience. The results showed that male participants with as well as without action video gaming experience performed significantly better than female participants. These results suggest that differences in haptic parallelity matching between males and females seem to be related to gender, rather than action video gaming experience. When performing the parallelity task visually, no significant differences were found between the three groups, corroborating earlier hypotheses that women are less able to ignore the bias of the egocentric hand-centered reference frame than men when haptically paralleling orientations.
\end{abstract}

\section{Keywords}

Haptic Spatial Perception, Egocentric, Allocentric, Gender, Visual-Haptic Matching, Action Video Gaming

\section{Introduction}

Research has shown that when male and female participants are asked to haptically make two bars parallel to each other, males perform significantly better 
than females, resulting in smaller deviations from parallelity in the former (Hermens et al., 2006; Kaas \& Van Mier, 2006; Kappers, 2003, 2007; Van Mier, 2013, 2016, 2019, 2020; Volcic et al., 2008; Zuidhoek et al., 2007). In this so-called haptic parallelity task (Kappers, 1999; Kappers \& Koenderink, 1999), blindfolded participants are instructed to rotate a test bar, using one of their hands, in the same orientation as the orientation of a reference bar that they feel with the other hand. It has been shown that humans are not very proficient in haptically paralleling both bars, as often rather large deviations have been observed in both male and female participants (e.g. Kappers, 2002, 2003; Kaas \& Van Mier, 2006; Van Mier, 2013, 2016, 2019, 2020), taking into account the above mentioned gender difference. When one has to parallel both bars, one can use either an allocentric or an egocentric reference frame, or a combination of both frames. When using an allocentric frame of reference, the orientation of the test bar is coded in relation to a frame external to and independent of the position of the perceiver. Allocentric referencing in the haptic parallelity task would result in veridical performance, with both bars being parallel. However, when using an egocentric reference frame, the test bar is oriented in relation to a frame internal to the perceiver. Considering that the deviations from parallelity are systematic and directed in the natural orientation of the hand, results from several studies support the suggestion that the egocentric reference frame is centered on the hand (Kappers \& Liefers, 2012; Kappers \& Viergever, 2006; Van Mier, 2013, 2016, 2019, 2020; Volcic \& Kappers, 2008; Volcic et al., 2009). The use of such a reference frame would result in rather large deviations from parallelity, with both bars having different orientations. Due to the fact that deviations in haptic parallelity matching are smaller than when only an egocentric reference would have been used, Kappers $(2002,2003)$ has proposed that participants most likely use a frame that is intermediate between allo- and egocentric referencing.

The deviations from parallelity are thought to be influenced by the bias of the hand-centered egocentric reference frame. The weighing of each frame can be manipulated by using different procedures and has been shown to be dependent on the gender of the participant (see Van Mier, 2014). Manipulations increasing the weight of the allocentric reference frame resulted in reduced deviations, with an effect that was similar in both men and women (Van Mier, 2013, 2016, 2019, 2020). This suggests that the observed gender difference is not due to an inability of women to use allocentric cues. In conditions of the parallelity task in which the test bar had to be rotated with the hand, significant gender difference were found (Hermens et al., 2006; Kaas \& Van Mier, 2006; Kappers, 2003, 2007; Van Mier, 2013, 2016, 2019, 2020; Volcic et al., 2008; Zuidhoek et al., 2007). Only when the motor response at the test bar was reduced (Van Mier, 2013) or eliminated (Kappers \& Schakel, 2011: in the visual condition; Van Mier, 2016) or when a bar had to be rotated to match a verbally stated clock time but no reference bar had to be felt (Zuidhoek et al., 2007), differences between the genders 
were not significant. Zuidhoek et al. (2007) speculated that women are most likely less able to overcome the bias of the egocentric reference frame of the hand. Results reported by Van Mier (2013, 2016, 2019, 2020) corroborate this line of thought.

In a recent study from our lab (Van Mier \& Jiao, 2020), we compared performance on haptic parallelity matching in male participants who did or did not play real time strategy (RTS) action video games with a so-called bird's eye view. These video games are played from a top-down perspective and promote an allocentric perspective (Dobrowolski et al., 2015; Kühn et al., 2014). Because we tested habitual RTS action video gamers with more than 5 year experience and playing at least a minimum of 5 hours per week, we hypothesized that this group would show enhanced allocentric processing resulting in smaller deviations in the haptic parallelity task than participants without action video gaming experience. That was indeed what we found; male action video gamers had significantly smaller deviations than males who did not or hardly played action video games (Van Mier \& Jiao, 2020). That brought up the question if the previously observed gender differences regarding haptic parallelity performance could be related to better allocentric processing in men due to the fact that in the general population the percentage of males playing action video games is much larger than of females. Terlecki et al. (2011) reported that of more than 2000 college students that filled out several psychological questionnaires, of which several questions were related to video game playing and experience, $74 \%$ of men and only $27 \%$ of women played videogames at the time of the survey, with women playing significantly less frequently than men. Women had been playing an average of 2 - 5 years while men had played an average of 10 years. A nationwide representative survey including almost 3000 German participants between the ages of 16 to 93 years, showed that in the group of frequent gamers, $70 \%$ of the players were male. Furthermore, male gamers preferred significantly more often game genres with action video game characteristics, like shooter and RTS games, than females (Rehbein et al., 2016). Focusing on competitive action video gaming, Ratan and colleagues (2015) found that of almost 17.000 players that responded to a survey addressing certain game characteristics, only $4 \%$ to $6 \%$ were female ( $2 \%$ did not report their gender). Based on over 270.000 gamers who completed a 5-minute survey to obtain a report of their gaming motivations (Gamer Motivation Profile), Yee (2017) reported that only 18.5\% of the gamers who took the survey were women. Looking at the different game genres, he found that only around $20 \%$ of those female gamers played some sort of action video game. Similar gender differences regarding frequency and game genre have been reported for adolescents. In a representative sample of more than 4000 American high school students aged between 14 to 18 years, $76 \%$ of boys indicated that they played video games at least 1 hour per week, while this percentage was 29 for girls (Desai et al., 2010). With more than 11.000 German adolescents, aged 13 to 18 years, being surveyed regarding video game depen- 
dency, Rehbein et al. (2015) found that boys played on average 162 min of video games per day compared to girls only playing $27 \mathrm{~min}$. In addition, Greenberg et al. (2010) stated that of more than 1200 children, adolescents, and students, male video gamers played on average 2 to 3 times the weekly average of female players. They also found that males played significantly more games that fell in the category of action video games. Taking these numbers and video game genres into account, one might argue that the observed gender differences in haptic parallelity matching might not be due to gender per se, but rather to enhanced spatial processing in males as a result of action video gaming. To establish if the aforementioned differences in haptic parallelity performance between males and females could be related to superior performance in males due to video gaming experience, the current study compared haptic parallelity performance of the above mentioned male participants who did or did not play action video games with performance on the same task in female participants without action video game experience. It was expected that female participants who didn't play action video games would have significantly higher deviations from parallelity than male participants who did play these games. If we would find that male and female participants without gaming experience would have similar deviations, then the observed differences might be related to something else than gender. However, if the former would have smaller deviations than the latter, the observed differences would be most likely related to gender and not to experience with action video gaming.

Previous research on parallelity matching has shown that gender differences were absent in conditions were participants had full view of the set-up and/or test hand (Kappers \& Schakel, 2011: in the visual condition; Van Mier, 2013, 2016, 2020). The current study therefore also compared performance between the groups in a version of the parallelity task in which participants had full view of the set up, and could use allocentric cues, like the sides of the plates with the bars, the table, walls, doors etc. In this condition, participants looked at the orientation of the reference bar and only used their (test) hand to parallel this orientation on the test bar. Because the egocentric bias of the hands plays a minor role in this condition, it was expected that the groups would show similar deviations in this visual-haptic version of the parallelity task.

\section{Method}

\subsection{Participants}

Data from thirty participants, which all had normal or corrected-to-normal vision, 20 males and 10 females, were analyzed to address the above mentioned research question. Participants were divided into three groups. The first group consisted of 10 male participants, who were habitual action video gamers (mAVG), the second group included 10 males, who had little or no experience with action video games (mNAVG), while 10 female participants without any action video game experience made up the third group (fNAVG). No female 
action video gamers were included, because it is hard to find women with sufficient action video game experience, as has been reported by others (Green et al., 2012). The male participants were the same participants as reported in our previous study (Van Mier \& Jiao, 2020). Eight of the female participants had participated in our 2020 study (Van Mier, 2020). We were able to retroactively obtain data regarding video game experience of eight of the female participants from that study, of whom none had played action video games before or at the time of testing. Two additional female participants without action video gaming experience were tested for the current study. Ages in the first group (mAVG) ranged from 19 to 44 years, with a mean age of 23.8 years $(\mathrm{SD}=7.4)$, in the second group (mNAVG) from 19 to 55 years, with a mean of 25.1 years $(\mathrm{SD}=11.3$ ), and in the third group (fNAVG) from 19 to 46 years, with a mean age of 24.7 years $(\mathrm{SD}=7.7)$. There was no significant difference regarding age between the groups $(F(2,27)=0.055, p=0.95)$. Annett's hand preference questionnaire was used to assess handedness (Annett, 2004). Two participants from the first group and one from the second group were left handed. Most of the participants were students at Maastricht University, the others were recruited among (gaming) friends. Participants received a monetary voucher, with students having the additional option to choose course credits instead of the monetary reward. All participants gave written informed consent prior to the study, which had been approved by the ethics review committee of the Faculty of Psychology and Neuroscience of Maastricht University and was performed in line with the ethical standards of the Helsinki Declaration of 1964.

Male participants who had played action video games for at least 5 years with a minimum of 5 hours per week and were still playing at the time of testing, made up the first group (mAVG). They played on average 9.5 hours (SD = 8.6) per week, and all played real-time strategy (RTS) action video games. Male participants in the second group (mNAVG) played no RTS video games and only three participants played sometimes other genre action video games for up to 2 hours a week for a short period (average 0.3 hours per week, $S D=0.6$ ). As stated before, none of the female participants in the third group (fNAVG) played action video games before or at the time of testing. Differences between the 3 groups regarding action video game experience as determined by number of play hours per week were significant $(F(2,27)=11.795, p<0.001)$. Post hoc comparisons with Bonferroni correction showed that mAVGs played significantly more hours than mNAVGs and fNAVGs (both $p=0.001$ ), while the difference between male and female non-players was not significant $(p=1.0)$.

\subsection{Materials}

Participants were asked to fill out the Video Game Experience Survey of Terlecki and Newcomb (2005). Based on this survey the total hours of video gaming per week were established as well as the kind and genre of video games that a participant played. For a more detailed description regarding the use of this survey, 
see Van Mier and Jiao (2020). Based on this survey it was established that all mAVGs played RTS action video games, while none of the other participants played this kind of games or played regularly other kinds of action video games.

Parallelity matching performance was measured using two square metal plates of 30 by $30 \mathrm{~cm}$ with an aluminum bar positioned in the middle of the plate. Each plate was covered with a plastic layer on which a protractor was printed (see Figure 1). The protractor had a radius of $10 \mathrm{~cm}$. Each bar was $20 \mathrm{~cm}$ in length and had a diameter of $1.1 \mathrm{~cm}$ and a small arrow-shaped point at one end of the bar, enabling accurate setting and reading of the reference and test bar of about $0.5^{\circ}$. In the middle of the bar a small pin was attached that slotted in a small hole in the center of the protractor, making rotation of the bar possible.

To achieve the setting of precise orientations, two magnets were attached to the bottom on the test bar increasing resistance to accidental movements and avoiding over- and undershooting. The reference bar had two additional magnets, to make sure participants would not accidentally rotate this bar. The boards were placed on anti-slip mats to avoid movement or displacement of the boards. To avoid that participants would see the protractors in the visual-haptic block and would parallel both bars based on information from the protractors, they were covered with a circular black paper (see Van Mier, 2020; Van Mier \& Jiao, 2020). By lifting the edge of the paper just a little, the experimenter was able to easily set the orientation of the reference bar and read the orientation of the test bar without the participant seeing the protractor.

\subsection{Set-Up and Procedure}

Parallelity performance was measured during a haptic and a visual-haptic block. The haptic block was always performed first. During this block participants were blindfolded and were instructed to parallel the orientation of the reference bar,

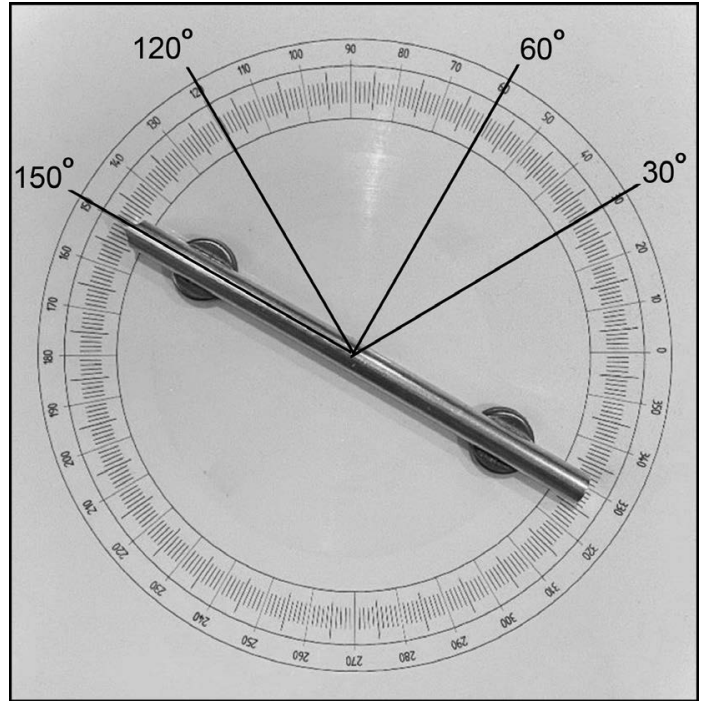

Figure 1. The protractor showing the test bar and the four orientations used in the study. 
which they felt with their non-dominant hand, on the test bar, which they had to rotate with their dominant hand. Both hands had to be placed simultaneously on the bars. During the visual-haptic block, participants had full view of the set-up and were told to look at the orientation of the reference bar without touching the bar and to parallel this orientation on the test bar using their dominant hand. In this block participants could both see and feel the orientation of the test bar. In both blocks, participants were instructed to place their stretched hand(s) on the bar(s) with their middle finger resting on the bar. This instruction was given to make sure that differences in deviations between haptic and visual-haptic performance would not be related to differences in hand position at the test bar. A significant difference in deviations in parallelity performance due to a change in hand position at the test bar has been reported in one of our previous studies (Van Mier, 2019).

For reasons of variation in stimulus presentation, the following four different reference orientations were used in the study: $30^{\circ}, 60^{\circ} 120^{\circ}$ and $150^{\circ}$ (see Figure 1). To prevent that participants would recognize cardinal orientations of $0^{\circ}$ and $90^{\circ}$ in the visual-haptic trials and use the sides of the plates and/or table to align the test bar to, these orientations were not used to be paralleled. They were only used as starting positions for the test bar with the side of the bar with the arrow being directed either upwards or to the right. All four orientations were presented with each of the two starting positions. The order and repetition of the orientations was randomized within each block and for each participant, taking into account that the same orientation was never presented consecutively. For the current study the results of one haptic and one visual-haptic block were used.

After filling out and signing the consent form, participants were tested regarding their understanding of parallelity by instructing them to line up two pens in such a way that they were parallel to each other using different orientations. During the experiment participants were seated in front of the boards, which were placed on a table at an equal distance from the midline of the participant's body. The distance between the centers of the boards was approximately twice the length of the arm. Arm length was measured from the top of the shoulder to the beginning of the hand and the distance between the plates was assessed by subtracting $3 \mathrm{~cm}$ from the arm length to ensure a comfortable position of arms and hands for each orientation (see also Van Mier, 2019). The mean distance between the plates was therefore $91.0 \mathrm{~cm}$ for mAVGs, $92.0 \mathrm{~cm}$ for mNAVGs and $87.4 \mathrm{~cm}$ for fNAVGs. This difference in distance between the groups was not significant $(F(2,27)=1.342, p=0.28)$. Most participants performed 6 haptic and 5 visual-haptic blocks, which were presented alternately. However, because we are interested in gender differences in pure haptic and visual-haptic parallelity, for the current study we only take into account performance during the first haptic and first visual-haptic block. All participants started with the haptic block. Because they were blindfolded during this block, the experimenter positioned the participant's hands just above the bars, and in- 
structed them to feel the reference bar as well as rotate the test bar with their stretched whole hand. The same instruction regarding the position of the hand was given in the visual-haptic block, with the exception that participants just looked at the reference bar without touching it and only rotated the test bar. After the haptic block, participants took off the blindfold so they had full view during the visual-haptic block. They were, however, asked to close their eyes between trials when the experimenter changed the orientation of the reference bar for the next trial.

\subsection{Statistical Analysis}

The dependent variable was the deviation between the orientation of the reference bar and the orientation of the test bar. For right-handed participants deviations clockwise to the reference bar were noted as positive values, and deviations counterclockwise to the reference bar as negative values. This was reversed for left-handed participants, with clockwise deviations being noted as negative and counterclockwise as positive. Deviations were averaged over the two repetitions and four orientations. A repeated measurement ANOVA was performed with Condition (2: haptic vs visual-haptic) as independent within factor and Group (3: $\mathrm{mAVG}, \mathrm{mNAVG}$, and $\mathrm{ANAVG}$ ) as independent between factor. Separate analyses were performed on the deviations in the haptic and visual-haptic block. Partial eta-squared $\left(\eta_{p}^{2}\right)$ was used to calculate effect sizes for condition and group (all 3 groups). When comparing male and female non-gamers directly, Cohen's $d$ was used to measure effect size. The Shapiro-Wilk test was used to test for normality because of the small sample size. Normality was obtained in all groups in the haptic block, as well as for the non-gamers in the visual-haptic block. Normality was not obtained for the video gamers in the visual-haptic block $(p=0.036)$. This was due to one video gamer, having larger deviations than the other gamers in this block. Levene's tests showed homogeneity of error variances for all groups for the haptic and visual-haptic block. For post hoc comparisons Bonferroni correction was used.

\section{Results}

We first performed a repeated measurement ANOVA including performance of the haptic and visual-haptic trials. This analysis showed a significant effect of condition $\left(F(2,27)=265.40, p<0.000, \eta_{p}^{2}=0.91\right)$ due to much larger deviations in the haptic trials (mean $=37.5^{\circ}, \mathrm{SD}=11.5$ ) than in the visual-haptic trials (mean $\left.=10.6^{\circ}, \mathrm{SD}=5.9\right)$. There was a significant effect of group $(F(2,27)=$ 22.592, $\left.p=0.001, \eta_{p}^{2}=0.63\right)$, as well as a significant interaction of group * condition $\left(F(2,27)=9.013, p=0.001, \eta_{p}^{2}=0.40\right)$. Differences between the groups were much larger in the haptic block than in the visual-haptic block (see Figure 2). Pairwise comparisons with Bonferroni correction showed significant differences between the three groups with $\mathrm{mAVG}$ having smaller deviations than mNAVGs $(p=0.002)$ and fNAVGs $(p<0.000)$, as well as mNAVGs having 


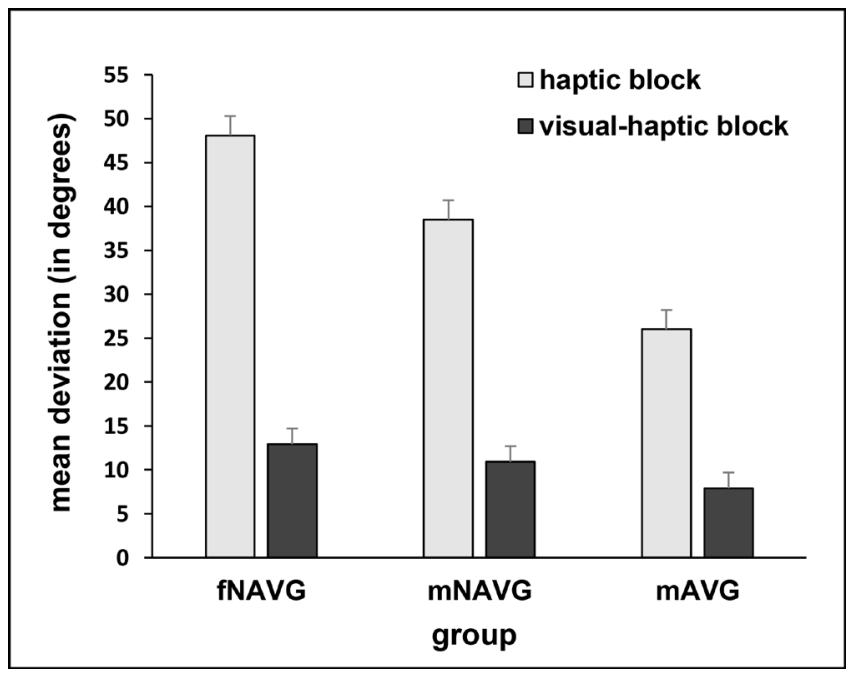

Figure 2. Mean deviations and standard error bars in the haptic and visual-haptic block for fNAVGs (female Non Action Video Gamers), mNAVGs (male Non Action Video Gamers), and mAVGs (male Action Video Gamers).

smaller deviations than fNAVGs $(p=0.023)$. Because of the significant interaction of group and condition, we additionally performed two separate analyses for the haptic and visual-haptic block.

\subsection{Haptic Parallelity Performance}

Focusing on the haptic trials, we found a significant effect of group $(F(2,27)=$ 24.78, $p<0.000, \eta_{p}^{2}=0.65$ ). Male action video gamers (mAVG) had a mean deviation of $26.0^{\circ}(\mathrm{SD}=8.0)$ when haptically paralleling both bars, while male (mNAVG) and female (fNAVG) non-video gamers had deviations of $38.5^{\circ}$ (SD $=7.6)$ and $48.1^{\circ}(\mathrm{SD}=5.2)$ respectively. Pairwise Bonferroni corrected comparisons showed significant differences between the three groups with mAVGs having significantly smaller deviations than mNAVGs $(p=0.001)$ and ANAVGs $(p<0.000)$, as well as mNAVGs having significantly smaller deviations than fNAVGs $(p=0.016)$. A direct comparison between male and female non-gamers showed that their performance was significantly different $(F(1,18)=10.87, p=$ $0.004)$. Cohen's $d$ was 1.20 , showing a large effect size of group. Because 3 participants in the mNAVG group played occasionally action video games while none of the females in the fNAVG group did, which could have skewed the results, performance of the 10 female non-gamers was compared to performance of the 7 male non-gamers. This analysis still showed a significant difference $(F(1.15)=9.45, p=0.008$ ), with an effect size of 1.31 (Cohen's $d$ ).

\subsection{Visual-Haptic Parallelity Performance}

Attending to the visual-haptic trials, we observed that the effect of group was not significant $\left(F(2,27)=1.96, p=0.16, \eta_{p}^{2}=0.13\right)$. Male action video gamers (mAVG) had a mean deviation of $7.9^{\circ}(\mathrm{SD}=6.2)$ when visually paralleling both 
bars, while male (mNAVG) and female (fNAVG) non-video gamers had deviations of $10.9^{\circ}(\mathrm{SD}=4.8)$ and $12.9^{\circ}(\mathrm{SD}=5.9)$ respectively. Pairwise Bonferroni corrected comparisons showed no significant differences between the three groups also not when comparing male gamers (mAVGs) with female non-gamers (fNAVGs) $(p=0.18)$.

We also tested to what extent the deviations in the visual-haptic trials were different from veridical performance, so from $0^{\circ}$ and we found that this difference was significant $\left(F(1,27)=103.922, p<0.001, \eta_{p}^{2}=0.79\right)$. The interaction with group was not significant $\left(F(2,27)=1.96, p=0.16, \eta_{p}^{2}=0.13\right)$.

\section{Discussion}

The current study addressed if previously observed gender differences in haptic parallelity matching could be related to enhanced performance in males due to the fact that males more often play action video games. Playing action video games has been found to result in better allocentric spatial performance in male participants (Van Mier \& Jiao, 2020). In the current study deviations from parallelity were compared between male action video gamers as well as male and female non-video gamers. As previously reported, male action video gamers had significantly smaller deviations in the haptic condition than male non-gamers (Van Mier \& Jiao, 2020). As expected, in the current study the former also performed significantly better than female non-gamers. Interestingly, we found that male non-gamers also outperformed female non-gamers. Although the sample size in the groups was small, the rather large effect size that was measured when deviations of male and female non-gamers were statistically compared, points towards a reliable effect. The fact that having to make two bars haptically parallel differentially modulated performance in male and female participants without action video gaming experience with the former outperforming the latter converges with numerous other studies reporting better haptic parallelity performance in men than in women (Hermens et al., 2006; Kaas \& Van Mier, 2006; Kappers, 2003, 2007; Van Mier, 2013, 2016, 2019, 2020; Volcic et al., 2008; Zuidhoek et al., 2007). As hypothesized, no significant gender differences were found in the visual-haptic condition, replicating earlier findings that including informative vision abolished the gender effect (Kappers \& Schakel, 2011: in the visual condition; Van Mier, 2013, 2016, 2020).

Because three participants in the male non-gamer group occasionally played action video games, while none of the females played these games, one could argue that the significant performance differences between the male and female non-gamers might have been skewed by performance of those three occasional gamers. However, when we compared only the seven male participants who had no action video game experience at all with the female non-gamers, the male non-gaming participants still had significantly smaller deviations than the female non-gaming participants. A large effect size was also found for this comparison. So even males without any video gaming experience performed better 
than the females in the current study, suggesting a gender difference with respect to haptic parallelity matching rather than performance differences between the genders due to any action video gaming experience.

The above-mentioned results regarding haptic parallelity matching suggest that previously reported gender differences are not (solely) due to differences between the genders related to action video gaming. Previous studies researching the effect of action video gaming have also shown significant differences in performance between male and female non-gamers (Feng et al., 2007; Boot et al., 2008). The former reported significant differences between the percentage of correct scores on a Useful Field of View (UFOV) task between gamers and non-gamers. In the non-gamer group, the difference in scores between male and female participants was also significant, with male non-gamers outperforming female non-gamers (Feng et al., 2007). Boot and colleagues (2008) measured accuracy in a Functional Field of View (FFOV) task in gamers and non-gamers. Although no significant difference in scores was found between gamers and non-gamers, a direct comparison between male and female non-gamers showed that males performed significantly better than females in this group (Boot et al., 2008). The field of view task assesses the capacity of visual attention and its spatial distribution in the visual modality (Green \& Bavelier, 2003). These gender related results on the field of view task might suggest that the performance differences between male and female non-gamers in this task could be related to differential spatial performance in both genders, which might explain the gender differences in the haptic parallelity task. However, as already stated in the introduction, gender differences in haptic parallelity performance were not significant when allocentric processing was stimulated and the motor response with the test hand was eliminated (Van Mier, 2016; Kappers \& Schakel, 2011: visual condition) or reduced (Van Mier, 2013). Apparently, men and women use allocentric referencing to process spatial orientations in these versions of the parallelity task in a more or less similar way. This is in line with other studies reporting similar performance in men and women in spatial orientation tasks (Bosco et al., 2004) and gender differences being mainly related to spatial tasks that involve spatial working memory (Voyer et al., 2017). Furthermore, results have shown that females selected an ego- or allocentric strategy in equal proportions as males in studies addressing ego- and allocentric strategy choices in navigation tasks (Van Gerven et al., 2012; Livingstone-Lee et al., 2014).

The findings of the current study suggest that other factors than spatial ability might explain the observed gender differences in the haptic parallelity task. One of these factors is most likely the fact that it is harder for women to ignore the egocentric bias of the hand, as proposed by Zuidhoek and colleagues (2007). This is supported by findings of Kappers (2007) who reported that the relative contribution of ego- and allocentric referencing is most likely different for men and women. She found that women had larger egocentric weighing factors than men. If this is indeed the case, we would expect that female action video gamers 
would still have larger deviations than male gamers, but smaller than female non-gamers, and maybe similar deviations as male non-gamers. This could be addressed in a follow-up of the current study including also habitual female action video gamers.

When participants in the current study were able to see both bars during the visual-haptic trials, the groups performed at a similar level. In this condition participants can use allocentric cues, like the sides of the plates and/or table to parallel the bars. Although the test hand had to be used to rotate and set the test bar, the reference bar was not felt with the other hand. The egocentric bias when trying to align both hands, like in the haptic trials, was therefore abolished. This is in line with results reported by Zuidhoek and colleagues (2007) who found similar performance for male and female participants in a condition in which they were asked to only use one hand to orient a bar to a verbally stated clock time. Overcoming this aligning of the hands seems to be especially difficult for women, as indicated by results of other studies (Zuidhoek et al., 2007; Kappers \& Schakel, 2011; Van Mier, 2013, 2016, 2019, 2020). The fact that no gender differences were found in the visual-haptic condition is consistent with the afore-mentioned explanation regarding similar performance for both genders in spatial orientation tasks in which the bias of the hand-centered egocentric reference frame is reduced or eliminated. In the current study participants only used their test hand to match the orientation of the test bar to the orientation of the reference bar. One might have expected more or less veridical performance in the visual-haptic trials where participants had full view of the set-up. However, as has been shown by others, parallelity in visual space is also prone to distortions (Cuijpers et al., 2000, 2003; Kappers \& Schakel, 2011; Liu \& Ando, 2018). It has been suggested that this is most likely due to the bias of an eye-/head-centered egocentric reference frame (Kappers \& Schakel, 2011).

A limitation of the current study is its correlational nature. We did not test non-gamers after training on action video games. It was assumed that gamers would perform better in the haptic parallelity task because of their enhanced spatial processing, especially improved allocentric processing, due to playing RTS action video games. Although a causal link has been shown between action video gaming and enhanced spatial attention and processing in the visual modality (e.g. Bediou et al., 2018; Spence \& Feng, 2010), making it likely that such a link might also exist for the haptic domain, a causative relationship has not yet been established in the haptic modality. It might be that the video gamers in the current study performed better than the non-gamers because of a population bias. It is possible that people who have better spatial and/or allocentric processing are more attracted to and/or spend more time playing RTS action video games. Furthermore, one cannot rule out that it is not the RTS game genre that resulted in improved performance in haptic parallelity matching but playing action video games in general. The latter have been shown to improve not only visual processing but also auditory processing (Green et al., 2010), as well as to en- 
hance multisensory perception (Donohue et al., 2010). Considering the fact that numerous studies have demonstrated that action video game playing has shown transfer effects beyond the activities that are trained in these video games (Boot et al., 2011; Bavelier et al., 2018; Spence \& Feng, 2010), it is plausible that this also entails to haptic processing. However, only a direct causal relationship between action video gaming and enhanced performance in haptic parallelity matching can support such an explanation. A direct comparison between haptic parallelity performance of players of RTS games and players of other action video game genres, like first shooter video games, could be set up to examine if the action video game genre plays a differential role in haptic parallelity performance.

Although the current study was not set up to directly address clinical/social or practical implications, the increasing development of techniques like robotics and virtual reality and the use of mobile devices and haptic displays have augmented an interest in haptic perception. Therefore, enhancing fundamental knowledge about processes that play a role in haptic perception is important and necessary (Kappers \& Bergmann Tiest, 2013). Furthermore, in situations in which visual information is not available or cannot be used one has to rely on haptic information. Gaining understanding and being aware about the limitations of our haptic spatial system and having knowledge about strategies to reduce these limitations (Van Mier, 2014, 2019) can be of great importance when performing tasks in which visual information is not or less available.

\section{Conclusion}

The present study replicated previously reported performance differences between males and females in haptic parallelity matching. Additionally, significant differences in haptic parallelity performance were found between male and female non-gamers. The latter result suggests that the haptic performance differences are most likely gender-related and do not appear to be associated with action video gaming experience. When performing the same task visually, males and females performed at a similar level. Evidence showing that gender differences are mainly found when an orientation that is haptically felt by one of the hands has to be paralleled haptically using the other hand, supports the proposal that women are less able to ignore the biasing influence of the hand-centered egocentric reference frame when setting two bars parallel to each other haptically.

\section{Acknowledgements}

The author would like to thank Laura Finn and Hui Jiao for their help in the data collection.

\section{Conflicts of Interest}

The author declares no conflicts of interest regarding the publication of this paper. 


\section{References}

Annett, M. (2004). Hand Preference Observed in Large Healthy Samples: Classification, Norms and Interpretations of Increased Non-Right-Handedness by the Right Shift Theory. British Journal of Psychology, 95, 339-353. https://doi.org/10.1348/0007126041528130

Bavelier, D., Bediou, B., \& Green, C. S. (2018). Expertise and Generalization: Lessons from Action Video Games. Current Opinion in Behavioral Sciences, 20, 169-173. https://doi.org/10.1016/j.cobeha.2018.01.012

Bediou, B., Adams, D. M., Mayer, R. E., Tipton, E., Green, C. S., \& Bavelier, D. (2018). Meta-Analysis of Action Video Game Impact on Perceptual, Attentional, and Cognitive Skills. Psychological Bulletin, 144, 77-110. https://doi.org/10.1037/bul0000130

Boot, W. R., Blakely, D. P., \& Simons, D. J. (2011). Do Action Video Games Improve Perception and Cognition? Frontiers in Psychology, 2, 226. https://doi.org/10.3389/fpsyg.2011.00226

Boot, W. R., Kramer, A. F., Simons, D. J., Fabiani, M., \& Gratton, G. (2008). The Effects of Video Game Playing on Attention, Memory, and Executive Control. Acta Psychologica, 129, 387-398. https://doi.org/10.1016/j.actpsy.2008.09.005

Bosco, A., Longoni, A. M., \& Vecchi, T. (2004). Gender Effects in Spatial Orientation: Cognitive Profiles and Mental Strategies. Applied Cognitive Psychology, 18, 519-532. https://doi.org/10.1002/acp.1000

Cuijpers, R. H., Kappers, A. M. L., \& Koenderink, J.J. (2000). Large Systemic Deviations in Visual Paralellelism. Perception, 29, 1467-1482. https://doi.org/10.1068/p3041

Cuijpers, R. H., Kappers, A. M. L., \& Koenderink, J.J. (2003). The Metrics of Visual and Haptic Space Based on Parallelity Judgements. Journal of Mathematical Psychology, 47, 278-291. https://doi.org/10.1016/S0022-2496(03)00004-X

Desai, R. A., Krishnan-Sarin, S., Cavallo, D., \& Potenza, M. N. (2010). Video-Gaming among High School Students: Health Correlates, Gender Differences, and Problematic Gaming. Pediatrics, 126, e1414-e1424. https://doi.org/10.1542/peds.2009-2706

Dobrowolski, P., Hanusz, K., Sobczyk, B., Skorko, M., \& Wiatrow, A. (2015). Cognitive Enhancement in Video Game Players: The Role of Video Game Genre. Computers in Human Behavior, 44, 59-63. https://doi.org/10.1016/j.chb.2014.11.051

Donohue, S. E., Woldorff, M. G., \& Mitroff, S. R. (2010). Video Game Players Show More Precise Multisensory Temporal Processing Abilities. Attention, Perception, \& Psychophysics, 72, 1120-1129. https://doi.org/10.3758/APP.72.4.1120

Feng, J., Spence, I., \& Pratt, J. (2007). Playing an Action Video Game Reduces Gender Differences in Spatial Cognition. Psychological Science, 18, 850-855. https://doi.org/10.1111/j.1467-9280.2007.01990.x

Green, C. S., \& Bavelier, D. (2003). Action Video Game Modifies Visual Selective Attention. Nature, 423, 534-537. https://doi.org/10.1038/nature01647

Green, C. S., Pouget, A., \& Bavelier, D. (2010). Improved Probabilistic Inference as a General Learning Mechanism with Action Video Games. Current Biology, 20, 1573-1579. https://doi.org/10.1016/j.cub.2010.07.040

Green, C. S., Sugarman, M. A., Medford, K., Klobusicky, E., \& Bavelier, D. (2012). The Effect of Action Video Game Experience on Task-Switching. Computers in Human Behavior, 28, 984-994. https://doi.org/10.1016/j.chb.2011.12.020

Greenberg, B. S., Sherry, J., Lachlan, K., Lucas, K., \& Holmstrom, A. (2010). Orientations to Video Games among Gender and Age Groups. Simulation \& Gaming, 41, 238-259. 
https://doi.org/10.1177/1046878108319930

Hermens, F., Kappers, A. M. L., \& Gielen, S. C. A. M. (2006). The Structure of Frontoparallel Haptic Space Is Task Dependent. Perception \& Psychophysics, 68, 62-75. https://doi.org/10.3758/BF03193656

Kaas, A. L., \& Van Mier, H. I. (2006). Haptic Spatial Matching in Near Peripersonal Space. Experimental Brain Research, 170, 403-413. https://doi.org/10.1007/s00221-005-0223-7

Kappers, A. M. L. (1999). Large Systematic Deviations in the Haptic Perception of Parallelity. Perception, 28, 1001-1012. https://doi.org/10.1068/p281001

Kappers, A. M. L. (2002). Haptic Perception of Parallelity in the Midsagittal Plane. Acta Psychologica, 109, 25-40. https://doi.org/10.1016/S0001-6918(01)00047-6

Kappers, A. M. L. (2003). Large Systematic Deviations in a Bimanual Parallelity Task: Further Analysis of Contributing Factors. Acta Psychologica, 114, 131-145.

https://doi.org/10.1016/S0001-6918(03)00063-5

Kappers, A. M. L. (2007). Haptic Space Processing-Allocentric and Egocentric Reference Frames. Canadian Journal of Experimental Psychology, 61, 208-218. https://doi.org/10.1037/cjep2007022

Kappers, A. M. L., \& Bergmann Tiest, W. M. (2013). Haptic Perception. Wiley Interdisciplinary Reviews: Cognitive Science, 4, 357-374.

https://doi.org/10.1002/wcs.1238

Kappers, A. M. L., \& Koenderink, J. J. (1999). Haptic Perception of Spatial Relations. Perception, 28, 781-795. https://doi.org/10.1068/p2930

Kappers, A. M. L., \& Liefers, B. J. (2012). What Feels Parallel Strongly Depends on Hand Orientation. In P. Isokosi, \& J. Springare (Eds.), Haptics: Perception, Devices, Mobility, and Communication, Volume 7282 of Lecture Notes on Computer Science (pp 239-246). Berlin: Springer. https://doi.org/10.1007/978-3-642-31401-8 22

Kappers, A. M. L., \& Schakel, W. B. (2011). Comparison of the Haptic and Visual Deviations in a Parallelity Task. Experimental Brain Research, 208, 467-473. https://doi.org/10.1007/s00221-010-2500-3

Kappers, A. M. L., \& Viergever, R. F. (2006). Hand Orientation Is Insufficiently Compensated for in Haptic Spatial Perception. Experimental Brain Research, 173, 407-414. https://doi.org/10.1007/s00221-006-0377-y

Kühn, S., Gleich, T., Lorenz, R. C., Lindenberger, U., \& Gallinat, J. (2014). Playing Super Mario Induces Structural Brain Plasticity: Gray Matter Changes Resulting from Training with a Commercial Video Game. Molecular Psychiatry, 19, 265. https://doi.org/10.1038/mp.2013.120

Liu, J., \& Ando, H. (2018). Response Modality vs. Target Modality: Sensory Transformations and Comparisons in Cross-Modal Slant Matching Tasks. Scientific Reports, 8, Article No. 11068. https://doi.org/10.1038/s41598-018-29375-w

Livingstone-Lee, S. A., Zeman, P. M., Gillingham, S. T., \& Skelton, R. W. (2014). Navigational Strategy May Be More a Matter of Environment and Experience than Gender. Learning and Motivation, 45, 30-43. https://doi.org/10.1016/j.lmot.2013.09.003

Ratan, R. A., Taylor, N., Hogan, J., Kennedy, T., \& Williams, D. (2015). Stand by Your Man: An Examination of Gender Disparity in League of Legends. Games and Culture, 10, 438-462. https://doi.org/10.1177/1555412014567228

Rehbein, F., Kliem, S., Baier, D., Mößle, T., \& Petry, N. M. (2015). Prevalence of Internet Gaming Disorder in German Adolescents: Diagnostic Contribution of the Nine DSM-5 Criteria in a State-Wide Representative Sample. Addiction, 110, 842-851. 
https://doi.org/10.1111/add.12849

Rehbein, F., Staudt, A., Hanslmaier, M., \& Kliem, S. (2016). Video Game Playing in the General Adult Population of Germany: Can Higher Gaming Time of Males Be Explained by Gender Specific Genre Preferences? Computers in Human Behavior, 55, 729-735. https://doi.org/10.1016/j.chb.2015.10.016

Spence, I., \& Feng, J. (2010). Video Games and Spatial Cognition. Review of General Psychology, 14, 92-104. https://doi.org/10.1037/a0019491

Terlecki, M. S., \& Newcombe, N. S. (2005). How Important Is the Digital Divide? The Relation of Computer and Videogame Usage to Gender Differences in Mental Rotation Ability. Sex Roles, 53, 433-441. https://doi.org/10.1007/s11199-005-6765-0

Terlecki, M., Brown, J., Harner-Steciw, L., Irvin-Hannum, J., Marchetto-Ryan, N., Ruhl, L., \& Wiggins, J. (2011). Sex Differences and Similarities in Video Game Experience, Preferences, and Self-Efficacy: Implications for the Gaming Industry. Current Psychology, 30, 22-33. https://doi.org/10.1007/s12144-010-9095-5

Van Gerven, D. J., Schneider, A. N., Wuitchik, D. M., \& Skelton, R. W. (2012). Direct Measurement of Spontaneous Strategy Selection in a Virtual Morris Water Maze Shows Females Choose an Allocentric Strategy at Least as Often as Males Do. Behavioral Neuroscience, 126, 465-478. https://doi.org/10.1037/a0027992

Van Mier, H. I. (2013). Effects of Visual Information Regarding Allocentric Processing in Haptic Parallelity Matching. Acta Psychologica, 144, 352-360.

https://doi.org/10.1016/j.actpsy.2013.07.003

Van Mier, H. I. (2014). Haptic Perception of Parallelity. Psychology and Behavioral Sciences, 3, 212-221. https://doi.org/10.11648/j.pbs.20140306.16

Van Mier, H. I. (2016). Reducing the Motor Response in Haptic Parallel Matching Eliminates the Typically Observed Gender Difference. Experimental Brain Research, 234, 105-115. https://doi.org/10.1007/s00221-015-4437-Z

Van Mier, H. I. (2019). Changing the Influence of the Egocentric Reference Frame Impacts Deviations in Haptic Parallelity Matching. Experimental Brain Research, 237, 2387-2395. https://doi.org/10.1007/s00221-019-05596-x

Van Mier, H. I. (2020). Combining Visual and Haptic Practice Significantly Reduced Deviations in Haptic Parallelity Matching. Acta Psychologica, 203, Article ID: 103008. https://doi.org/10.1016/j.actpsy.2020.103008

Van Mier, H. I., \& Jiao, H. (2020). Influence of Action Video Gaming on Spatial Representation in the Haptic Modality. Experimental Brain Research, 238, 2769-2781. https://doi.org/10.1007/s00221-020-05931-7

Volcic, R., \& Kappers, A. M. L. (2008). Allocentric and Egocentric Reference Frames in the Processing of Three-Dimensional Haptic Space. Experimental Brain Research, 188, 199-213. https://doi.org/10.1007/s00221-008-1353-5

Volcic, R., Van Rheede, J. J., Postma, A., \& Kappers, A. M. L. (2008). Differential Effects of Non-Informative Vision and Visual Interference on Haptic Spatial Processing. $E_{X}$ perimental Brain Research, 190, 31-41. https://doi.org/10.1007/s00221-008-1447-0

Volcic, R., Wijntjes, M. W., \& Kappers, A. M. L. (2009). Haptic Mental Rotation Revisited: Multiple Reference Frame Dependence. Acta Psychologica, 130, 251-259. https://doi.org/10.1016/j.actpsy.2009.01.004

Voyer, D., Voyer, S. D., \& Saint-Aubin, J. (2017). Sex Differences in Visual-Spatial Working Memory: A Meta-Analysis. Psychonomic Bulletin \& Review, 24, 307-334. https://doi.org/10.3758/s13423-016-1085-7

Yee, N. (2017). Beyond 50/50: Breaking down the Percentage of Female Gamers by Ge- 
nre. https://quanticfoundry.com/2017/01/19/female-gamers-by-genre

Zuidhoek, S., Kappers, A. M. L., \& Postma, A. (2007). Haptic Orientation Perception: Sex Differences and Lateralization of Functions. Neuropsychologia, 45, 332-341.

https://doi.org/10.1016/j.neuropsychologia.2006.05.032 